\title{
Norois
}

Environnement, aménagement, société

$244 \mid 2017$

Sport, immigration, urbanisation littorale, climat sahélien, biodiversité

\section{Le vieillissement des personnes immigrées en Maine-et-Loire}

Analyse du discours d'acteurs médico-sociaux, associatifs et politiques The aging of immigrants in Maine-et-Loire. Analysis of the discourse of medico-social, political and community actors

\section{Aurélien Martineau et Mathilde Plard}

\section{(2) OpenEdition}

\section{Journals}

Édition électronique

URL : https://journals.openedition.org/norois/6154

DOI : $10.4000 /$ norois. 6154

ISBN : 978-2-7535-7420-5

ISSN : $1760-8546$

\section{Éditeur}

Presses universitaires de Rennes

\section{Édition imprimée}

Date de publication : 30 décembre 2017

Pagination : 19-33

ISBN : 978-2-7535-7418-2

ISSN : 0029-182X

\section{Référence électronique}

Aurélien Martineau et Mathilde Plard, « Le vieillissement des personnes immigrées en Maine-et-Loire », Norois [En ligne], 244 | 2017, mis en ligne le 30 décembre 2019, consulté le 13 janvier 2022. URL : http://journals.openedition.org/norois/6154 ; DOI : https://doi.org/10.4000/norois.6154 


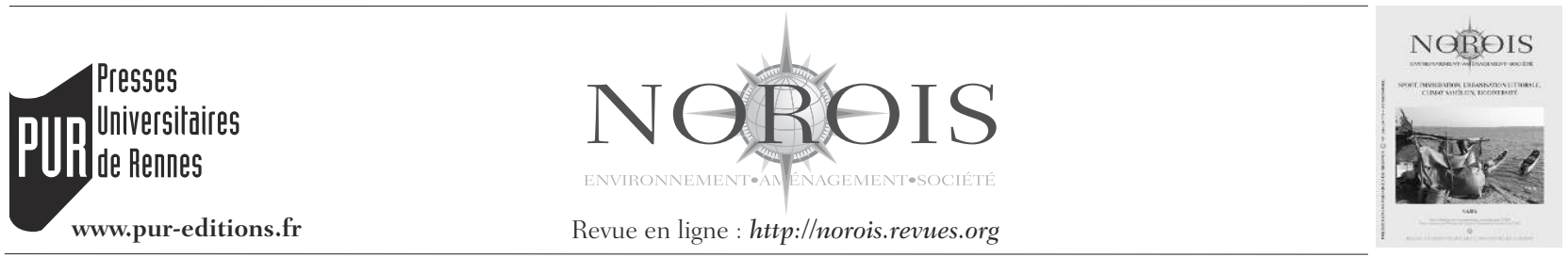

\title{
Le vieillissement des personnes immigrées en Maine et Loire Analyse du discours d'acteurs médico-sociaux, associatifs et politiques
}

\author{
The Aging of Immigrants in Maine-et-Loire \\ Analysis of the Discourse of Medico-social, Political and Community actors
}

\author{
Aurélien Martineau ${ }^{\text {a*}}$, Mathilde Plard ${ }^{\mathrm{b}}$ \\ * Auteur correspondant : Tel : +33 (0)6 89180063 \\ a UMR 6590 CNRS ESO Angers, Université d'Angers, Maison de la Recherche Germaine-Tillion - 5 bis boulevard \\ Lavoisier, 49045 Angers Cedex 1, France. (aurelien.martineau@univ-angers.fr) \\ b UMR 6590 CNRS ESO Nantes, Université de Nantes, Campus Tertre - Chemin de la Censive-du-Tertre, \\ BP 81227, 44312 Nantes Cedex 3, France. (mathilde.plard@univ-nantes.fr)
}

Résumé : Cet article analyse les conditions de vieillissement des personnes âgées immigrées en Maine-et-Loire à partir d'un corpus d'entretiens réalisé auprès 35 acteurs médico-sociaux, associatifs et politiques sur le département. La finalité de l'étude est de comprendre quels sont les enjeux et les problématiques que peuvent rencontrer les immigrés en vieillissant au sein d’un département où leur présence démographique est moindre. En conclusion, trois principaux enjeux ressortent : le maintien des pratiques transmigratoires face à l'altération de l'état de santé, le moindre recours à l'offre gérontologique associé à l'importance de l'aide informelle et enfin les disparités territoriales que peuvent connaître les immigrés âgés (offre de service ou d'action à leur attention et (in)formation des professionnels).

\begin{abstract}
This article analyzes the aging conditions of elderly immigrants in the Maine-et-Loire department, in western France, based on a corpus of interviews conducted with 35 social and gerontological actors throughout the department. This study seeks to understand what is at stake and the problems that immigrants can encounter as they age in an area where their demographic presence is reduced. In conclusion, three main issues result from this analysis: the continuance of trans-migratory practices in the face of deteriorating health; a reduction in the use of gerontological services combined with the prominence of informal aid; and the territorial disparities that elderly immigrants may encounter (range of services and actions available to them; level of training and knowledge of professionals).
\end{abstract}

Mots clés : immigré âgé, vieillissement - santé - acteurs sociaux

Keywords: elderly immigrants - aging - health - social actors

\section{INTRODUCTION}

À l'échelle de la France comme au niveau mondial, le vieillissement démographique est l'une des problématiques majeures du $\mathrm{XxI}^{\mathrm{e}}$ siècle (Dumont, 2005). L'augmentation de l'espérance de vie asso- ciée à la croissance de la population âgée génère des enjeux politiques, sociaux et gérontologiques sans précédent.

Sur le territoire français, les situations et les expériences du vieillir sont diversifiées, les profils de la population âgée sont multiples. Dans ce contexte, 
les personnes immigrées vieillissent aussi en France à l'instar de la population autochtone. En 2013, les personnes immigrées âgées de 55 ans ou plus étaient près de 1886000 , soit $9,5 \%$ des 55 ans et plus en France. Bien que la population immigrée ne connaisse pas un vieillissement démographique, du fait du renouvellement des flux de migrants, un phénomène de gérontocroissance est à l'œuvre. Les récents rapports menés par l'Assemblée nationale (Jacquat et Bachelay, 2013) et par diverses institutions (Rafaï et al., 2007; Soumeur-Méreau, 2014) révèlent l'acuité des enjeux qui entourent l'avancée en âge de ces publics et leur arrivée au seuil du grand-âge.

Sur le plan démographique, la répartition géographique des migrants âgés à travers la France est inégale et des disparités importantes s'observent selon leur plus ou moins grande présence au sein des départements et régions françaises. La surreprésentation des migrants âgés sur certains territoires est le témoin du passé industriel de la France et dépend également de la proximité géographique avec les pays d'origine. Dans les territoires où les migrants âgés sont peu nombreux, il n'en reste pas moins qu'ils vieillissent aussi. Dès lors, différents enjeux existent dans leur possibilité d'accès au bienêtre dans l'avancée en âge.

Cet article s'intéresse précisément aux conditions de vieillissement des personnes âgées immigrées dans le Maine-et-Loire. Ce département est particulièrement éclairant puisque comme de nombreux départements, la population immigrée y est faiblement représentée au sein de la population âgée. En 2013, 2,8 \% de la population âgée de 55 ans et plus du département était immigrée, soit 6624 personnes. Le Maine-et-Loire représentait l'un des départements français où la proportion d'immigrés dans la population âgée était la plus faible. D'où l'intérêt d'interroger les problématiques et enjeux que rencontrent les aînés immigrés dans leur avancée en âge.

La littérature scientifique et professionnelle fait état de l'existence d'une méconnaissance mutuelle entre les personnes immigrées et les services gérontologiques et sociaux (Bartkowiak, 2008). Face à ce manque de connaissance, il semble important de documenter les situations d'immigrés âgés auprès des acteurs du secteur médico-social. Ces derniers sont souvent les premiers à intervenir dans la mise en place d'accompagnements sociaux (en termes de recours aux droits notamment). Pour répondre à ce besoin de connaissance, une étude a été réalisée dans le Maine-et-Loire pour étudier les situations des personnes immigrées âgées. En complément de l'analyse des données démographiques et contextuelles, une série d'entretiens a été menée auprès des services gérontologiques et sociaux. Des propos d'élus, de professionnels et de directeurs d'établissements ou de services médico-sociaux, d'intervenants de collectivités territoriales, de centres sociaux et des bénévoles associatifs ont été recueillis. L'analyse de contenu des différents entretiens met en lumière les problématiques rencontrées au quotidien par ces personnes dans l'avancée en âge à partir du regard et des retours d'expérience des acteurs.

Cet article est structuré autour de deux parties. Le contexte territorial du Maine-et-Loire ainsi que la méthodologie employée sont d'abord présentés. Les résultats de l'analyse des discours des acteurs sont développés ensuite.

\section{CONTEXTE TERRITORIAL ET MÉTHODOLOGIE}

\section{Personnes âgées immigrées, quelles situations en Maine-et-Loire?}

Le Maine-et-Loire est un département de l'ouest de la France. Recensant un peu plus de 800000 habitants, il se structure autour d'une grande agglomération (Angers) et deux villes de tailles moyennes (Cholet et Saumur). À l'image de la France, ce département est lui aussi confronté au vieillissement de sa population. Le phénomène y apparait néanmoins limité, il compte parmi les départements vieillissant le moins : $78^{\text {e }}$ en 2013 selon l'indice de vieillissement. La part des personnes âgées de 65 ans et plus dans sa population $(17,5 \%)$ reste moins importante que la moyenne en France métropolitaine (19,7\%) (figure 1). Le vieillissement démographique représente essentiellement un enjeu d'avenir puisque les projections départementales de l'INSEE font état de l'augmentation à venir de la population âgée. En 2040, la proportion des 65 ans et plus dans l'ensemble de la population augmenterait de près de 7 points de pourcentage (de $18 \%$ en 2014 à $25 \%$ en 2040).

À l'échelle départementale, le vieillissement concerne également les personnes immigrées 
Figure 1 : Part de la population âgée de 65 ans et plus par département en 2013 en France Distribution of the population over the age of 65 by département in 2013 in France

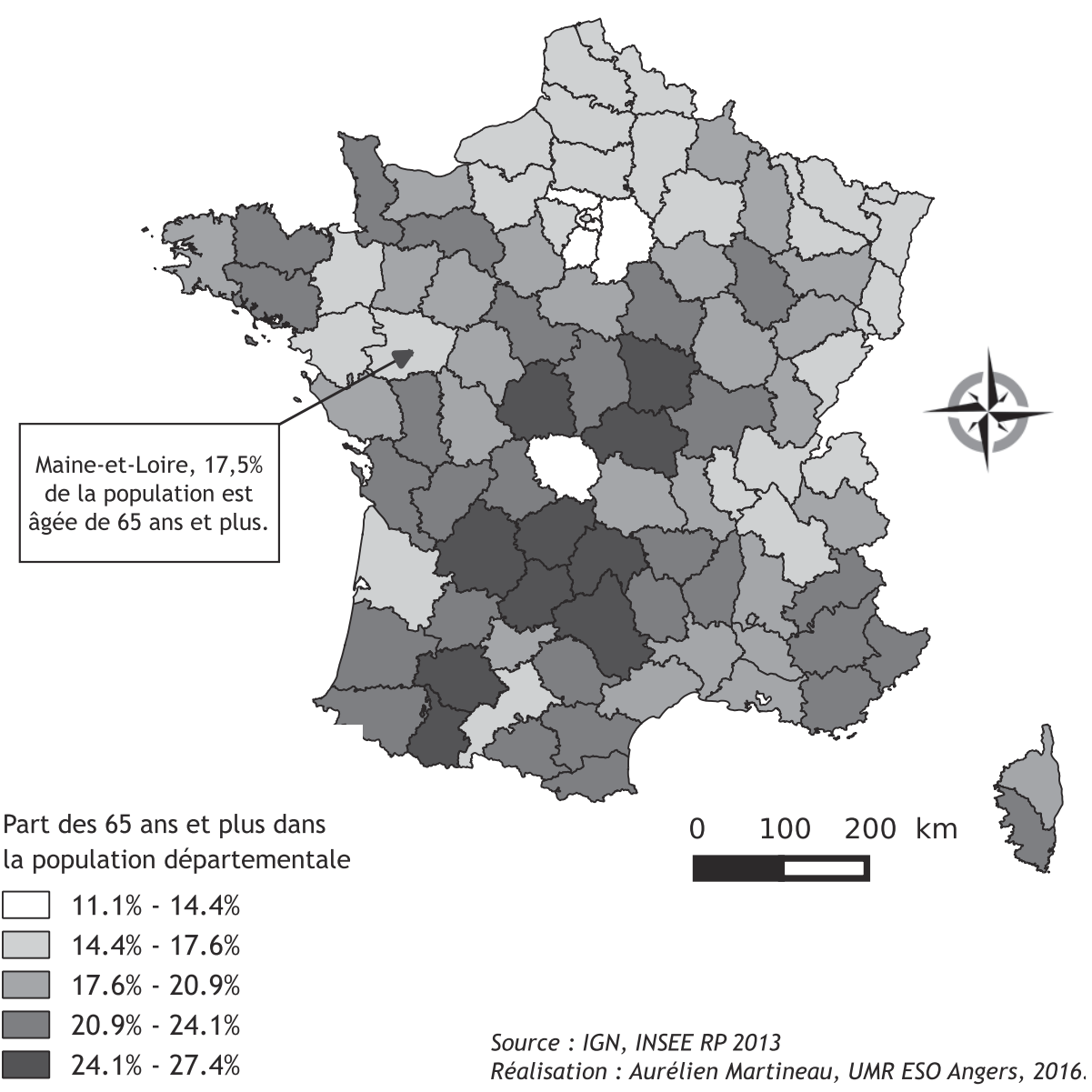

âgées, leur nombre étant en augmentation. En 2013, ce sont 6624 personnes immigrées de 55 ans ou plus qui résidaient en Maine-et-Loire (tableau 1), sur un peu moins de 30000 personnes immigrées. Elles représentaient 2,8\% de la population départementale âgée de 55 ans et plus, ce qui témoigne de leur faible représentation démographique. À titre de comparaison, dans le département de la Seine-Saint-Denis, les personnes immigrées de 55 ans et plus représentaient en 2013 près de $35 \%$ de l'ensemble de la population de cette même classe d'âge.

La distribution des personnes immigrées en France apparaît donc inégale et la moindre présence de la population immigrée sur le département s'explique par différents facteurs historiques et géographiques. Pour l'historien Marais (2016) de l'association Histoire et Mémoire de l'Immigration en Anjou, le Maine-et-Loire n'a pas pris part aux principaux mouvements migratoires connus dès la

\begin{tabular}{|c|c|c|c|c|}
\hline Département & Population & $\begin{array}{c}\text { Nb d'immigrés dans la } \\
\text { population des 55 ans et } \\
\text { plus }\end{array}$ & $\begin{array}{c}\text { Part d'immigrés dans la } \\
\text { population des 55 ans et } \\
\text { plus }\end{array}$ & $\begin{array}{c}\text { Classement départemen- } \\
\text { tal : part d'immigrés dans } \\
\text { la population des 55 ans } \\
\text { et plus }\end{array}$ \\
\hline Cantal & 147035 & 994 & $1,7 \%$ & $96^{\mathrm{e}}$ \\
\hline Maine-et-Loire & 800191 & 6624 & $2,8 \%$ & $86^{\mathrm{e}}$ \\
\hline Seine-Saint-Denis & 1552482 & 118459 & $35,1 \%$ & $1^{\text {er }}$ \\
\hline
\end{tabular}

Tableau 1 : Population immigrée âgée en 2013, comparaison départementale (Source : INSEE RP 2013 - Réalisation : A. Martineau, UMR ESO, Université d'Angers, 2017)

Elderly immigrant population in 2013 - comparison by département 
Seconde Guerre mondiale. Le département « rattrape son retard à partir de 1962 : de 1962 à 1982, la population étrangère a augmenté de 169 \% en France, de $539 \%$ en Maine-et-Loire! Le Maine-etLoire prend en marche le train de l'immigration : à cette date, ce sont les Portugais et les Marocains qui arrivent en France, et donc dans ce département » (Marais, 2016). La présence de la population immigrée âgée se caractérise actuellement par le contexte historique (origines des migrants étant arrivés durant la période précédemment citée, souvent pour une immigration de travail, un regroupement familial ou en tant que réfugié politique), et au regard des populations arrivées plus récemment. Sur le plan géographique, il apparaît par ailleurs que la population immigrée est inégalement répartie. En 2013, plus de 6 personnes immigrées sur 10 résidaient au sein de l'une trois principales villes de Maine-et-Loire que sont Angers, Cholet et Saumur. Ces trois villes représentant $29 \%$ de la population départementale. Constat similaire pour la population immigrée âgée de 55 ans et plus, près d'une personne sur deux habitait en 2013 à Angers (2 064 personnes) ou Cholet (1 193 personnes) (tableau 2 et figure 2).

La population immigrée sur le Maine-et-Loire est moins âgée (23\% est âgée de 55 ans et plus) que la population non immigrée $(29 \%)$. Quant à l'origine des immigrés âgés selon le pays de naissance, près d'une personne sur deux était née en Europe et près d'un tiers dans l'un des pays du Maghreb (figure 3). Des nuances existent selon les territoires et les villes. Pour exemple, à Cholet, $41 \%$ des personnes immigrées de 55 ans et plus étaient nées en Afrique contre $61 \%$ pour Angers.

Les personnes immigrées âgées représentent donc une faible proportion de la population départementale. En Maine-et-Loire, leurs situations sont peu documentées et seule une référence a été observée au sein d'un rapport du Conseil de développement de la région d'Angers sur le vieillissement de la population (une page de synthèse a été produite par une association locale sur « la situation particulière des immigrés vieillissants " (Conseil de développement de la région d'Angers 2012). De même, comme une majorité des conseils départementaux, l'insertion de la question des personnes âgées immigrées aux documents directeurs de l'action départementale (schéma gérontologique, schéma unique) se fait rare (Jacquat et Bachelay, 2013) et n'apparaît pas dans le département.

\section{Terrains d'étude, acteurs et méthodologie}

Pour Pian (2014), la moindre visibilité quantitative des immigrés âgés dans certains départements ne fait pas pour autant disparaître les enjeux qu'ils rencontrent dans l'avancée en âge. «L'intérêt d'un phénomène social ne se résume pas à son importance quantitative. Au contraire, le fait que les immigrés soient proportionnellement peu nombreux sur un territoire donné soulève des problématiques particulières au niveau de l'accès aux soins d'une partie de cette population » (Pian, 2014). L'absence d'étude antérieure dans le Maineet-Loire renforce la nécessité de porter un regard sur les situations rencontrées par les immigrés âgés qui peuvent être qualifiés de «vulnérables » dans la littérature scientifique (Samaoli, 2012). Pour mener à bien cette démarche, le choix a été fait de ne pas centrer cette recherche sur une population particulière (selon l'origine, le sexe ou les conditions d'habitat) mais de tendre à rester ouvert à l'ensemble des situations composant les immigrés âgés dans le département. Deux principales raisons justifient ce choix. Premièrement, le fait de ne pas

\begin{tabular}{|c|c|c|c|c|}
\hline Territoires & $\begin{array}{c}\text { Nombre } \\
\text { d'immigrés }\end{array}$ & $\begin{array}{c}\text { Part des immigrés dans la } \\
\text { population }\end{array}$ & $\begin{array}{c}\text { Nombre d'immigrés dans } \\
\text { la population des 55 ans } \\
\text { et plus }\end{array}$ & $\begin{array}{c}\text { Part des immigrés dans la } \\
\text { population des 55 ans et } \\
\text { plus }\end{array}$ \\
\hline Angers & 13227 & $8,8 \%$ & 2064 & $5,2 \%$ \\
\hline Cholet & 4341 & $8,1 \%$ & 1193 & $6,9 \%$ \\
\hline Saumur & 1106 & $4,0 \%$ & 312 & $3,1 \%$ \\
\hline Maine-et-Loire & 29127 & $3,6 \%$ & 6624 & $2,8 \%$ \\
\hline
\end{tabular}

Tableau 2 : Répartition de la population immigrée au sein des trois principales villes du Maine-et-Loire en 2013 (Source : INSEE RP 2013 - Réalisation : A. Martineau, UMR ESO, Université d'Angers, 2017)

Distribution of the immigrant population in the three main cities of Maine-et-Loire in 2013 
Figure 2 : Répartition de la population immigrée âgée de 55 ans ou plus par commune dans le Maine-et-Loire en 2012

Distribution of the immigrant population over the age of 55 by municipality in Maine-et-Loire in 2013

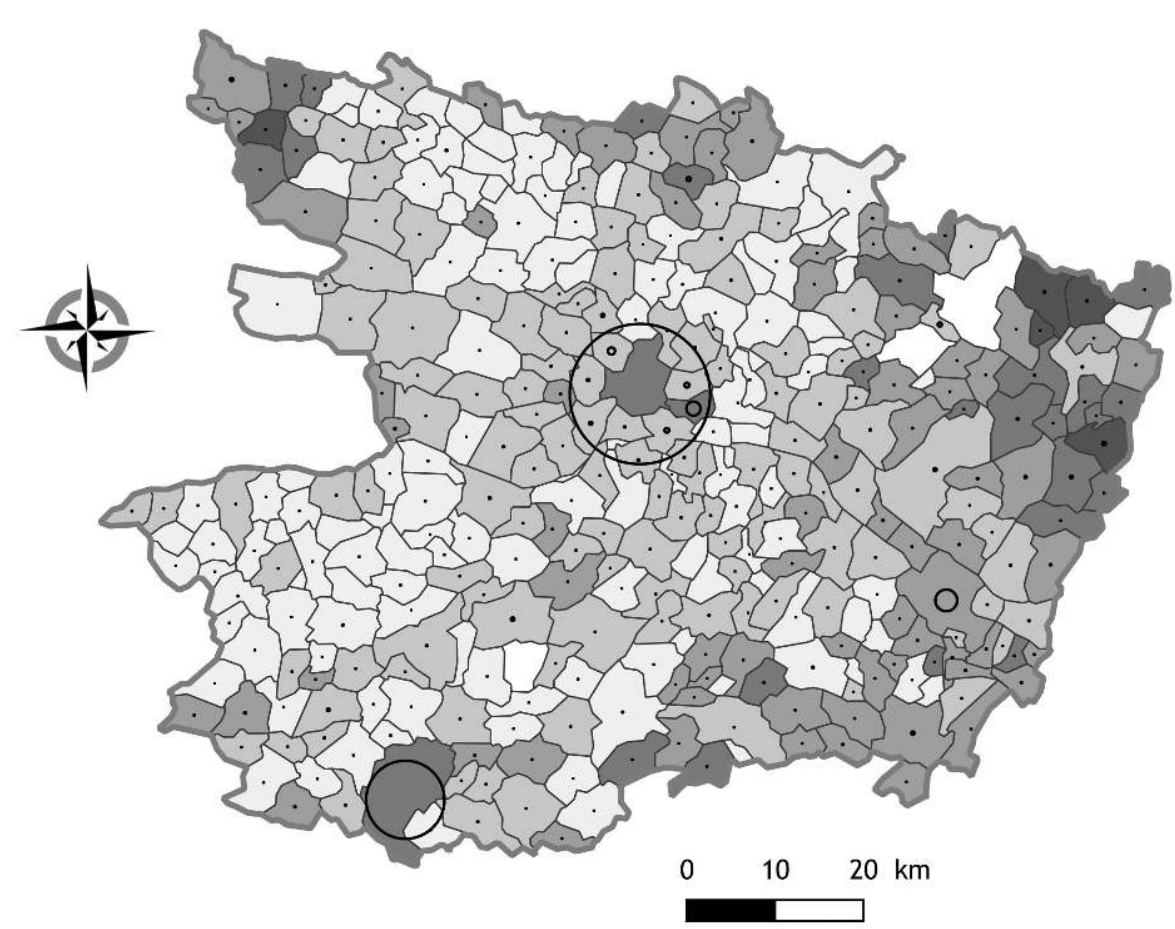

Part de la population immigrée dans l'ensemble de la population âgée de 55 ans ou plus par commune (\%)

$0.0-0.9$

$0.9 \cdot 2.3$

$2.3-4.7$

$4.7 \cdot 10.0$

$10.0-17.9$
Nombre de personnes immigrées âgées de 55 ans ou plus : 2008 (Angers)

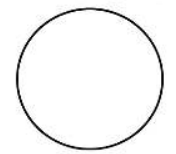

1119 (Cholet)

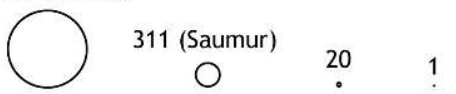

Source : IGN, INSEE RP 2012

Réalisation : A. Martineau, UMR ESO, Université d'Angers, 2017 se fermer à l'hétérogénéité sociale et culturelle des migrants âgés présents sur le département et donc de ne pas se focaliser uniquement sur un public qui serait perçu comme particulièrement vulnérable (pour exemple, les résidents de foyers de travailleurs migrants) ou une origine surreprésentée dans le département (les personnes originaires du Maghreb). Deuxièmement, c'est avant tout le fait d'avoir connu au cours de sa vie la migration qui est déterminant dans les expériences de vie de ces publics. Cette affirmation s'inscrit dans la continuité de ce qu'exprime la sociologue Attias-Donfut (2012) pour qui les personnes immigrées âgées ont avant tout en commun «leur mobilité internationale, et souvent des pratiques transnationales qui en font les agents d'importants échanges interculturels ». Le périmètre d'étude défini est centré sur les villes d'Angers et de Cholet au regard de la surreprésentation des personnes immigrées âgées en comparaison du reste du département.

Rencontrer et questionner les acteurs a permis d'élargir le spectre des situations d'immigrés âgés présentes sur le Maine-et-Loire à partir de l'ensemble des situations auprès desquelles ils ont pu intervenir. Les entretiens effectués ne constituent pas un échantillon statistique représentatif permettant de saisir l'ensemble des situations des immigrés âgés sur le département. Ceux-ci présentent avant tout l'intérêt de mettre à jour les interventions d'acteurs sociaux, gérontologiques, associatifs à travers les accompagnements et les actions qu'ils ont menés auprès de ces publics. Cela a pour avantage de mettre en lumière et de restituer les enjeux et les problématiques qu'identifient les acteurs dans les 


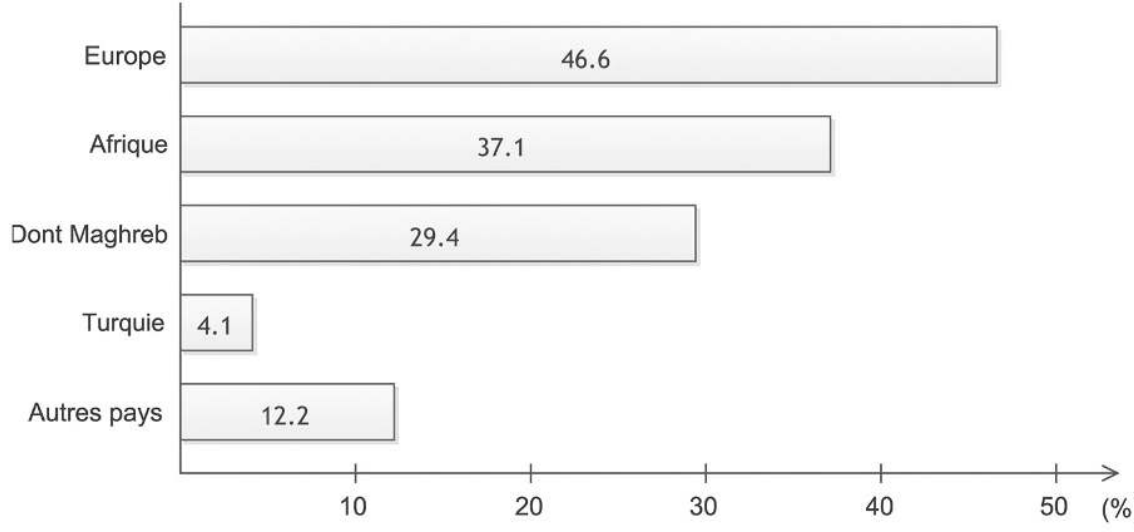

Source : INSEE RP 2013 - Réalisation : A. Martineau, UMR ESO, Université d'Angers, 2017.
Figure 3 : Répartition de la population immigrée âgée de 55 ans ou plus selon le pays de naissance en Maine-et-Loire en 2013

Distribution of the immigrant population over the age of 55 by country of birth in the Maine-et-Loire in 2013 situations de personnes âgées immigrées. Cela offre l'opportunité d'interroger également les ressources sociospatiales sur lesquelles les personnes peuvent ou non s'appuyer pour les soutenir dans l'avancée en âge. Ces entretiens permettent de restituer par ailleurs, les logiques, les représentations et les interrogations d'acteurs lorsqu'ils sont amenés à intervenir auprès de population minoritaire, voire invisible ou méconnue dans certains cas dans l'espace. Cependant, ils présentent également comme limites d'être bien souvent centrés sur les situations les plus complexes ou dans lesquelles les intervenants ont rencontré le plus de difficultés. Il est donc important de prendre une distance constructive avec les propos recueillis pour ne pas étendre ces constats à l'ensemble de la population immigrée âgée sur le département.

De mars à juillet 2016, 26 entretiens semi-directifs (dont 7 collectifs) ont été réalisés auprès de 35 professionnels, bénévoles et élus. 11 structures n'ont répondu que par échanges téléphoniques ou par emails. 45 structures n'ont pas répondu ou ont répondu défavorablement à la sollicitation dans le cadre de l'enquête (pour différents motifs : non-intérêt pour l'étude, non-intervention auprès du public, aucune disponibilité dans le délai proposé). Les entretiens ont été réalisés auprès d'institutions et acteurs diversifiés ${ }^{1}$.

Les entretiens ont été retranscrits et intégrés au logiciel d'analyse qualitative Nvivo. L'utilisation de cet outil numérique a permis de réaliser une analyse

1. Élus, Directions et intervenants en EHPAD, Services d'aide à domicile, Centres locaux de coordination gérontologique (CLIC), Centres de soins de suite et de longue durée (CSSLD), Foyers de travailleurs migrants et résidences sociales, Associations, Centres sociaux, Bailleur social. systématique du contenu des entretiens. L'analyse a consisté à mettre en œuvre un procédé de thématisation des fragments de discours identifiés dans chaque entretien. Ce procédé « constitue l'opération centrale de la méthode, à savoir la transposition d'un corpus donné en un certain nombre de thèmes représentatifs du contenu analysé. L'analyse thématique consiste, dans ce sens, à procéder systématiquement à l'examen discursif des thèmes abordés dans un corpus » (Paillé et Mucchielli, 2008). Cette démarche a permis de mettre en lumière les principaux thèmes, enjeux et problématiques identifiés par les acteurs lors des entretiens. Les résultats présentés sont uniquement valables au regard des contextes d'interventions des acteurs et des situations rencontrées dans un cadre territorial défini, ici le Maine-et-Loire.

\section{Analyse des Situations DES IMMIGRÉ(E)S ÂGES, RETOURS D'EXPÉRIENCES DES ACTEURS}

\section{La pluralité des situations : migration, origines et habitat}

Comme ailleurs les immigrés âgés du Maineet-Loire ne constituent pas un groupe homogène. Leurs parcours migratoires et leurs trajectoires de vie sont diversifiés. De «l'exil et le déchirement» (acteur ville d'Angers, 25) ${ }^{2}$ à l'enracinement pour reprendre le terme de la sociologue Attias-Donfut (2006), qui se traduit par « un attachement à la

\footnotetext{
2. Entre parenthèse la référence de la citation. La forme choisie $(1,2)$ : 1fonction et/ou institution de l'acteur; 2 - le numéro de la citation dans la base de données constituée.
} 
France, au pays dans lequel j'ai vécu » (élu Angers, 15), l'expérience de la vie dans le pays d'immigration peut être ressentie très différemment selon les situations rencontrées par les acteurs.

Warnes (2004) propose une typologie sur les migrants âgés en Europe pour décrire ces situations plurielles selon les motifs de la migration (raison professionnelle, familiale ou de convenance), les origines géographiques (européens/non-européens) et le sexe des migrants. Les situations rencontrées en Maine-et-Loire par les acteurs médico-sociaux, associatifs et politiques illustrent cette diversité. Seule une catégorie présente dans la typologie n'a pas été citée, celle des personnes ayant réalisé une migration sur le tard pour raison de convenance.

Pour les personnes dont l'arrivée en France est ancienne, souvent venues pour le travail, pour un regroupement familial ou en tant que demandeur d'asile, au temps de la retraite une part importante d'entre elles font le choix de s'installer durablement en Maine-et-Loire. Selon les acteurs, différents facteurs vont influer sur le choix de rester vivre en France à la retraite, avec pour une partie la poursuite de pratiques transmigratoires entre le pays d'accueil et le pays d'origine. La présence des enfants, l'offre médicale, l'absence prolongée du pays d'origine sont autant d'éléments qui, dans les situations, représentent des clés de compréhension quant aux choix de résidence des immigrés âgés. Pour certaines personnes, le fait de rester en France ne représente pas un choix, mais est contraint de par leur situation administrative. C'est le cas des personnes qui bénéficient de prestations sociales qui impliquent un temps de présence obligatoire sur le territoire français variant en fonction des dispositifs selon le principe de «territorialité » de la protection sociale (Math, 2012). Les personnes vivent alors une forme d'assignation à résidence sur le territoire français et ne peuvent retourner vivre définitivement dans leurs pays d'origine sous peine de perdre la totalité ou une partie des ressources octroyées par leurs droits sociaux.

Concernant les conditions de vie, les situations en matière d'habitat sont diverses, mais conformément aux données nationales (Croguennec, 2012), les immigrés vivent plus fréquemment au sein de l'habitat social. Sur la ville d'Angers, une responsable d'agence d'un bailleur social confirme leur présence dans le parc social et les probléma- tiques que cela peut générer notamment face au vieillissement et lors des opérations de rénovations/réhabilitations des logements sociaux. «Ce sont des personnes qui sont attachées à leur quartier et pour qui le projet d'un ailleurs en dehors de son quartier peut être difficilement envisageable, voire impossible» (bailleur social, 11). Les personnes immigrées résident également au sein du parc privé, en pavillon et sont également pour une partie propriétaire d'un bien au sein de leurs pays d'origine. Concernant les activités et les loisirs que les personnes peuvent avoir au temps de la retraite, les acteurs expriment qu'elles participent peu aux activités dédiées aux personnes retraitées sur le territoire. Les activités entre pairs d'origine, les jardins familiaux ou personnels, la religion sont pour nombre d'entre eux des activités centrales dans leur vie quotidienne. Autrement, il faut noter que certains publics apparaissent rencontrer davantage de difficultés dans la vie quotidienne en Maineet-Loire comparativement aux données de la littérature : les résidents âgés en foyer de travailleurs migrants et les femmes immigrées âgées (une partie ne parlant pas français et elles n'ont pas connu de socialisation professionnelle). Ces deux profils sont d'après les acteurs particulièrement exposés à diverses difficultés dans l'avancée en âge. Pour les résidents des structures d'ADOMA présents au sein de leur logement depuis de nombreuses années, ils ont majoritairement construit leur vie entre la France et leur pays d'origine. Sans famille ou celle-ci est présente au pays, avec de faibles ressources, l'avancée en âge peut être difficile et leur vie centrée autour du foyer et entre pairs. Pour les femmes, lorsqu'elles sont restées principalement au domicile familial, elles n'ont pu apprendre la langue et développer de réseaux hors du contexte familial et entre pairs, l'isolement ressenti peut être particulièrement intense. C'est ce que relève une action mise en œuvre par un centre socioculturel choletais au sein d'un quartier prioritaire. Pour l'animatrice de l'action, quand les femmes rencontrées, notamment maghrébines, ne parlent pas ou peu le français, n'ont pas le permis, cela limite leur déplacement et la réalisation des démarches quotidiennes indépendamment de leur mari ou de leurs enfants. "Elles vont dans très peu d'endroits, elles vont à la mosquée, au marché, au centre social et c'est tout, leurs déplacements se limitent à cela, 
elles ne voient pas autre chose et restent dans leur appartement, elles sont entre-elles » (Centre social Cholet, 11). Cet isolement a également été perçu auprès des personnes immigrées résidant sur l'un des principaux quartiers de la ville d'Angers, ce qui a abouti à la mise en place d'une action collective ${ }^{3}$ à leur attention pour créer un espace d'échange et de partage générateurs de liens sociaux. Ces deux groupes sont intéressants au regard des réponses apportées par les acteurs aux diagnostics effectués. Ils correspondent à des projets innovants répondant aux difficultés des personnes immigrées vieillissantes tels que les qualifie Wolff (2015) dans l'étude menée à l'échelle française auprès des professionnels du secteur sanitaire et social. Ces deux actions collectives proposent des sorties culturelles, touristiques, ouvertes sur les territoires de vie des personnes favorisant leurs découvertes de nouveaux lieux.

L'accès aux espaces dédiés aux personnes retraitées peut également être limité par les représentations qui sont associées à ces lieux de sociabilité, pourvoyeur de care face aux représentations de la piété filiale. À Angers, la coordinatrice du relai municipal sur le quartier de la Roseraie expliquait dans ce sens, que l'accès des personnes immigrées vieillissantes à la plateforme gérontologique de la ville (nommée Espace du bien-vieillir) peut être freiné par les habitants. Un travail de médiation a dû être amorcé pour déconstruire le fait que l'accès à cet espace ne signifie pas que les personnes immigrées n'obtiennent aucun soutien de la part de leur environnement familial. La coordinatrice donnait pour exemple la situation de personnes immigrées venues à l'espace gérontologique et interpellées par des habitants une fois à l'extérieur du bâtiment : "s'ils ressortent, quelqu'un leur dit, qu'est-ce qui se passe? C'est quoi? Tes enfants ils ne s'occupent pas de toi? Il y a un problème? » (Acteur ville d'Angers, 35). Ces exemples témoignent de l'importance d'interroger auprès de ces publics les pratiques tant sociales que spatiales qu'ils mettent en œuvre pour appréhender les contraintes et supports dont ils bénéficient au sein de leurs espaces de vie.

Les migrations tardives s'inscrivent quant à elles dans des situations de regroupement familial ou

3. [http://www.apriles.net/index.php?option=com_sobi2\&sobi2Task=sobi2 Details\&catid=4\&sobi2Id=1547\&Itemid=95] (consulté 12 avril 2016). pour des personnes qui font le choix de s'installer en France pour raison de santé. Dans le cadre du regroupement familial sur le tard, cela peut être mis en œuvre pour rejoindre un membre de la famille présent en France et qui du fait des enjeux associés au vieillissement a besoin d'une personne pour le soutenir. Une assistante sociale (AS) du CHU d'Angers fait ainsi part d'une femme africaine qui a rejoint son conjoint âgé de 80 ans en France qui a besoin d'aide au regard de sa situation médicale. Cette femme est «venue rejoindre son mari qui était en France depuis très longtemps. Elle l'aidait au quotidien et elle était épuisée [...] elle est arrivée il y a 6 mois, après 30 ans de mariage. Et pour elle, c'était vraiment compliqué d'arriver ici " (AS CHU Angers 14, 8). Quant aux personnes qui font le choix de venir pour raison de santé, sur Angers, les coordinatrices du CLIC sont intervenues auprès d'une dame âgée congolaise, ayant des problèmes de mobilité et des troubles cognitifs. Cette femme est «venue en France pour être soignée et elle n'est jamais repartie. [...] Le mari, il est resté au Congo. Donc elle vit avec la fille qui a 40 ans, des petits-enfants dans un logement qui n'est pas du tout prévu, tout petit, à l'étage sans ascenseur, elle dort dans la chambre de sa petite fille, sur un tout petit lit " (CLIC Angers, 24). Les services sociaux des Centres Hospitaliers confirment ce constat, des parents âgés ayant rejoint leurs enfants installés en France pour se faire soigner. Cependant, ces migrations tardives ne sont pas sans conséquence, car l'accès aux soins de santé peut être plus ou moins difficile en fonction des situations (assurance privée ou non, possibilité de bénéficier de l'aide médicale d'État). Les personnes peuvent parfois ne pas accéder aux soins dont elles auraient besoin.

\section{Grand âge, dispositifs gérontologiques et enjeux de la langue}

Les immigrés sont également confrontés aux enjeux du grand-âge et de la perte d'autonomie. La littérature s'accorde sur les difficultés particulières qu'ils rencontrent dans la vieillesse notamment au regard des parcours de vie qui ont pu fragiliser leur état de santé (Hamel et Moisy, 2013). Bien que ce dernier ne soit pas homogène, les personnes immigrées connaissent en moyenne une entrée en situation de dépendance plus précoce que le reste 
de la population (Jacquat et Bachelay, 2013). Nonrecours aux dispositifs, problème de la langue, isolement, précarité sont autant de facteurs qui complexifient le vieillissement prématuré de ces publics. Sur le département, les immigrés ne semblent pas échapper à ces constats. D’après les acteurs, ce qui diffère dans les interventions auprès des personnes immigrées comparativement au reste de la population concerne le recours aux services d'aide à la personne et aux établissements d'hébergement pour personnes âgées dépendantes (EHPAD). De nombreux retours vont dans ce sens et font part selon les origines et les situations de la difficile ou non-acceptation des interventions professionnelles.

Les facteurs avancés par les acteurs pour expliquer le faible recours aux services d'aide à domicile sont pluriels. Ces services peuvent ne pas correspondre aux attentes des personnes immigrées et être parfois refusés catégoriquement. Selon les situations, l'aide apportée aux personnes sera essentiellement du ressort de la famille. "Majoritairement c'est une prise en charge familiale donc c'est très rare qu'il y ait des interventions extérieures » (CSSLD Angers, 48). Les CLIC rencontrés confirment l'absence de demande, comme l'illustre la situation d'un «monsieur qui de par sa culture exprimait que c'était difficile qu'il y ait des gens qui interviennent à domicile donc il voulait tout faire tout seul et qu'il n'y ait pas des étrangers qui rentrent chez eux " (CLIC Angers, 2). Les CLIC expriment cependant être sollicités occasionnellement par les familles pour obtenir des informations sur la possibilité de rémunérer un aidant familial à partir de l'allocation personnalisée d'autonomie. Lorsque les personnes ne souhaitent recourir qu'à l'aide de la famille ou des proches, cela peut être complexe pour les intervenants notamment lorsque la situation médicale de la personne se dégrade et lors des retours d'hospitalisation. Au sein des résidences ADOMA du département, les immigrés âgés peuvent ainsi effectuer des allers-retours fréquents entre la résidence et l'hôpital, les solutions proposées pour soutenir les personnes n'étant pas acceptées par ces derniers. "Monsieur M. il refuse, il ne veut pas donc de toute façon, c'est vrai que des fois tu te dis, tu pourrais retravailler les retours à domicile pour justement éviter qu'il y en ait d'autres [des hospitalisations], pour les aides au repas, la toilette, exetera et c'est refusé. À un moment donné, cela tient un peu et puis cela relâche » (AS ADOMA, 41). Les habitudes alimentaires peuvent aussi représenter un frein aux interventions; comme dans l'exemple de cette femme âgée camerounaise pour qui «c'était extrêmement compliqué parce qu'elle avait sa façon de manger et ce n'était pas possible de cuisiner autrement [...] en travaillant tout ça, je me suis dit qu'il aurait presque fallu trouver une aide à domicile camerounaise qui aurait pu faire ce lien et cuisiner avec elle » (CLIC Angers, 16). Enfin, la barrière de la langue peut représenter une difficulté dans l'intervention, les professionnels ne maîtrisant pas forcément la langue étrangère parlée par la personne. C'est le cas, d'une auxiliaire de vie sociale intervenant auprès d'une femme âgée portugaise ne parlant pas le français. Pour pallier cette difficulté, l'intervenante s'est adaptée par la réalisation de pancartes en Portugais pour pouvoir communiquer avec la personne lorsque sa fille vivant avec elle était absente du domicile. Cet exemple illustre les stratégies d'adaptations mises en œuvre parfois dans les interventions professionnelles pour faire face aux contraintes rencontrées. En l'absence de solutions formalisées, l'appel au « bricolage » consistant «à travailler « avec les moyens du bord » (Wolff et Jovelin, 2015) est primordial pour s'assurer de la mise en œuvre des accompagnements sociaux et sanitaires des personnes âgées immigrées. L'ensemble de ces constats doit cependant être mis en perspective, l'ensemble des personnes immigrées ne refuse pas de recourir à l'intervention des services d'aide à domicile, mais leurs recours sont moindres par rapport au reste de la population à l'instar des résultats à l'échelle nationale (Croguennec, 2012). A contrario, les acteurs font part de l'acceptation sans appréhension des interventions des services infirmiers auprès des personnes immigrées pour bénéficier de soins à domicile. "Les soins on y arrive plus facilement [...] il y a la compréhension que tout le monde n'est pas infirmier, aide-soignant et que l'on ne peut pas faire n'importe quoi " (CLIC Loire, 52). Les retours ont été similaires concernant les EHPAD sur les territoires d'étude, les personnes immigrées n'y sont que très rarement présentes. Le conseil départemental de Maine-et-Loire rencontré par l'Observatoire National de l'Action Sociale (ODAS) dans le cadre d'une mission d'expertise ${ }^{4}$ sur les migrants âgés confirmait ce constat : « ce public est absent des établissements » (ODAS, 2014). Les

4. [http://odas.net/Groupe-de-travail-personnes-agees] (consulté 12 avril 2016). 
acteurs rencontrés font eux aussi référence à ces dimensions, proches des éléments développés précédemment. L'existence d'une «culture de la prise en charge familiale » chez une partie des immigrés âgés a pour conséquence de ne pouvoir envisager l'accueil en EHPAD d'un proche âgé. "C'est assez compliqué d'intervenir auprès de ce public d'origine étrangère qui a pour habitude une prise en charge familiale et qui n'attend pas l'EHPAD » (CLIC Angers, 8). Un représentant associatif exprime en ce sens que pour les personnes maghrébines, "il y a le poids de la culture morale. De mettre un parent dans une maison de retraite, c'est une sorte de bannissement social [...] c'est sacrilège, cela ne se fait absolument pas » (Association Angers, 19). Pour une partie des professionnels, l'accueil en EHPAD des immigrés n'est pas adapté au regard de divers enjeux qu'ils identifient : langue, habitudes alimentaires, pratiques religieuses, cohabitation avec d'autres résidents, préservation du lien avec le pays d'origine, etc. L'une des intervenantes en résidence ADOMA exprime que l'hébergement «en maison de retraite pour personnes âgées ce n'est pas $d u$ tout adapté non plus, du fait de leur mode de vie, fait d'aller-retour » (A.S. ADOMA, 45). Mêmes retours pour le CLIC d'Angers, dans l'une des interventions "c'était important de pouvoir proposer cette inscription en EHPAD. Mais en même temps, je me disais pour une personne qui ne s'exprime quasiment plus et qui ne comprend pas et qui ne parle pas français, cela va être très compliqué pour elle comme pour l'établissement " (CLIC Angers, 9). Pour autant, d'autres acteurs à l'image d'un directeur d'EHPAD sur Angers expriment que des adaptations sont possibles au sein des établissements. Le projet personnalisé de l'individu doit permettre de prendre en compte les éventuels besoins et de s'ajuster dans la mesure du possible aux attentes de la personne. L'accès limité aux EHPAD soulève aussi la question de leur accessibilité financière, bien que des dispositions existent (aide sociale à l'hébergement) pour soutenir les personnes. C'est ce qu’a également exprimé le conseil départemental lors de son audition par l'ODAS, le coût financier de l'hébergement en EHPAD étant un facteur explicatif de cette absence, en complément « d'un aspect [dit] culturel ». Le département précise qu'il observe des enjeux similaires au sujet des personnes non immigrées résidant en milieu rural. Ces derniers souhaitent plus fréquemment se maintenir à domicile et ont davantage de réticence à devoir quitter leur logement malgré l'inadaptation de celui-ci face aux difficultés rencontrées. L’offre gérontologique existante et la répartition des places disponibles en EHPAD habilité à l'aide sociale sur le territoire doivent également être considérées. Une assistante sociale d'un centre de soins de suite témoignait de la situation d'un homme portugais âgé pour qui l'entrée en EHPAD était envisagée, mais impossible à proximité de son lieu de résidence du fait de l'absence de places disponibles en EHPAD public : «quand on a parlé de l'institutionnalisation avec les enfants, la situation faisait que c'était possible, mais la proximité géographique avec leur père était primordiale. [...] c'est quasiment impossible d'avoir une place [sur Angers] ou alors il faut avoir des revenus importants. Là, les personnes quand elles ont des revenus, ça ne pose pas de problème mais sinon, ce n'est pas la même chose » (CSSLD-1, 7). Ces exemples illustrent que la problématique de l'accès des personnes immigrées aux EHPAD ne peut se résumer qu'aux contraintes culturelles comme facteur explicatif de leur faible ou non-recours aux institutions. Dans le cadre de l'enquête, moins d'une dizaine de situations ont été recensées au sein d'EHPAD publics. À l'image des exemples recueillis, l'entrée en établissement se met en œuvre parfois sous la contrainte, quand l'état de santé de la personne est trop dégradé ou que le proche aidant ne peut plus accueillir ou soutenir le parent. Dans l'exemple d'une dame âgée portugaise vivant avec sa fille, "les deux elles ne voulaient pas, elles ont toujours fait leur vie ensemble, puisqu'elles étaient veuves, elles habitaient ensemble donc il n'a pas été question de changer, il n'était pas question d'aller en maison de retraite. Elle est partie en structure quand ça n'allait plus du tout, avant non, non, non " (Aide à domicile Cholet, 7). Une aide-soignante intervenant au sein d'un EHPAD public accueillant des personnes immigrées déclarait qu'il existe aujourd'hui moins de barrières ou de freins pour s'ajuster aux besoins et envies des individus. Cependant, l'accueil en EHPAD des immigrés n'en reste pas moins difficile à certains égards, notamment quand il n'est pas possible de communiquer avec la personne ou lorsque des problèmes dans la cohabitation entre résidents existent, des situations de racisme ayant été rencontrées. Elle donne l'exemple d'une personne immigrée sur Cholet "c'est compliqué aussi pour lui parce qu'il est très typé et cela amène beaucoup de racisme, on a beaucoup de résidents qui ont 
80 ans qui ne supportent pas les personnes de couleurs différentes et lui en plus il ne sait pas trop bien parler le français, donc pour lui cela crée vraiment des difficultés » (Aide-soignante EHPAD, 8).

Enfin pour conclure, l'accueil des personnes immigrées peut se réaliser au sein de centre de soins de suite et de longue durée (CSSLD) et dans les services gérontologiques des Centres hospitaliers. Pour l'assistante sociale d'un CSSLD pour les personnes immigrées «à de rares exceptions, le maintien à domicile n'est plus possible et ils ont recours à de l'hospitalisation et ils n'arrivent pas à déboucher sur un autre projet que le retour à domicile même s'ils n'en peuvent plus » (AS CSSLD-2, 11). Pour cette intervenante, dans l'ensemble des situations rencontrées, la non (ou difficile) maitrise de la langue française représente une difficulté fréquente qui complexifie les interventions tant médicales que sociales auprès de ces publics : «Dans toutes les rares situations rencontrées, c'est le problème de la langue. [...] Souvent avec les pathologies liées aux troubles cognitifs, il y a un renforcement de l'utilisation de la langue maternelle et ça, c'est un problème pour les soins et pour travailler un projet » (AS CSSLD-2, 2). Au sein des services gérontologiques hospitaliers, les intervenants confirment les constats évoqués, les personnes immigrées représentant peu de situations. La prise en charge médicale reste la même que pour les non-immigrés, quelques différences s'observant dans la prise en charge selon les situations au regard de la culture, de la religion et des habitudes de vie. Au sein du CHU d'Angers, un cadre de santé dit prendre en charge deux ou trois situations de personnes immigrées sur un millier de patients par an, constat similaire à Cholet : "c'est des personnes auxquelles on a peu accès, j'en ai vu quelques-unes en gériatrie, en soins palliatifs, mais comme il y a une culture familiale qui est plus importante que nous [...] et bien au final ils ne sont pas très demandeurs an niveau des aides, cela se passe beancoup en famille» (AS CH Cholet, 4). D'après les professionnels, ces services disposent de moyens leur permettant de s'adapter tant vis-à-vis de l'alimentation (cuisine centrale) que pour communiquer avec les personnes (recours à des traducteurs, physiquement ou par téléphone). Les situations peuvent cependant sortir des prises en charge ordinaire et demandent alors des ajustements. C'est le cas de cette femme africaine atteinte de la maladie d'Alzheimer qui au sein de sa chambre souhaite dormir et manger par terre : "Ce qui se passe avec les personnes vieillissantes c'est qu'elles reprennent les habitudes de quand elles étaient jeunes, elles perdent la mémoire, tout ce qu'elles ont acquis récemment pour garder ce qui est de plus ancien et du coup les personnes immigrées, elles repartent sur leurs habitudes de vie et leurs coutumes de quand elles étaient enfant en Afrique » (Cadre santé, CHU Angers, 9). L'adaptation représente donc pour ces professionnels une aptitude essentielle pour intervenir et s'ajuster aux besoins des publics immigrés dans la vieillesse.

\section{La (trans)migration à l'épreuve de la vieillesse et de la mort}

Les liens qui unissent les personnes avec leur pays d'origine sont déterminants dans l'analyse des enjeux qui entourent leur avancée en âge. Les exemples de situations recueillies témoignent en effet des problématiques rencontrées par les personnes quand les pratiques et choix migratoires entrent en confrontation avec les difficultés rencontrées dans la vieillesse.

Les expériences de l'avancée en âge se poursuivent en effet dans la migration pour une partie des personnes immigrées, constamment en mouvement dans cet entre-deux composé des différents espaces de vie de l'individu. Après des années passées loin du pays d'origine, celui-ci a pu évoluer et le retour envisagé est parfois complexe. La distance qui s'est instituée avec son pays de naissance, parfois avec la famille ou les proches quand la personne a vécu seule en France, peut rendre les retours difficiles. Un responsable d'association exprime en ce sens «il y a de la rupture, quand on quitte un pays à 25 ans et qu'on reste plus de 40 ans, 50 ans ici, quel lien il reste? [...] Ils pourront vivre quelques mois, quelques semaines, mais pas toute l'année, pas toute la vie, ce n'est pas possible. Il y a trop de décalage»(Association 1 Angers, 19). Pour les individus qui ont vécu seuls en France, ces « célibataires géographiques » tels qu'ils sont nommés dans la littérature (Gallou, 2005), « quand ils partent au pays, ils n'ont jamais vécu avec leur famille donc ils ne savent pas quelle place ils ont, voilà c'est vraiment compliqué » (CLIC Cholet, 6). 
Autrement, pour l'ensemble des personnes immigrées, en fonction des pays d'origine, la mise en œuvre des pratiques transmigratoires peut être plus ou moins onéreuses et les voyages éprouvants. Une intervenante donne ainsi l'exemple d'une femme haïtienne qui ne peut plus retourner dans son pays d'origine. À l'inverse, des acteurs évoquent la situation d'une partie des personnes d'origine européenne qui mettent plus facilement en œuvre ses voyages et peuvent plus aisément envisager leur retraite dans leurs pays d'origine tout en maintenant des liens avec la France (si des enfants y sont présents par exemple) de par la proximité géographique. "Pour les personnes portugaises, c'est des personnes, ils ont conservé des maisons au Portugal donc c'est des personnes qui vont retourner vivre au Portugal après, en plus c'est moins loin techniquement que le Laos" (AS CH Cholet, 28). Quant à la réalisation des voyages, ceux-ci peuvent être épuisants dans l'avancée en âge comme dans l'exemple d'un homme immigré qui malgré ses 90 ans emprunte toujours le car pour se rendre au Maghreb (CLIC Cholet, 6). Certaines personnes immigrées âgées sont quant à elles dans l'impossibilité de réaliser les voyages au regard de leurs problèmes de santé. "Du fait de l'état de santé de Monsieur et bien ils sont bloqués là et donc on sent que ça c'est compliqué pour eux, de ne pas revoir, de ne pas retourner en Algérie, c'est difficile. Et pour elle aussi, puisque du coup elle s'interdit d'y retourner comme lui ne peut pas y retourner» (AS CSSLD, 37). De même, les problèmes de santé peuvent subvenir pendant ou à l'issue des voyages. Plusieurs acteurs font référence à des situations d'hospitalisation en urgence à la descente de l'avion. C'est le cas d'une femme âgée immigrée qui « a pris l'avion toute seule et deux fois de suite elle a été directement hospitalisée après l'avion. Elle arrive et claque à l'Hôpital donc deux fois de suite on a été interpellé dans ce sens-là " (CLIC Loire, 25). Ces complications durant les voyages peuvent donc mettre à mal la situation médicale, mais aussi parfois administrative des personnes immigrées. Selon le CLIC d'Angers, il y a des « personnes âgées qui partaient pendant plusieurs mois et qui pouvaient tomber malade dans son pays donc pas la possibilité de revenir et tous les droits à la retraite suspendus »(CLIC Angers, 22). Ces quelques exemples témoignent des enjeux inhérents aux pratiques transmigratoires au temps de la retraite et dans la vieillesse.

Mais ces enjeux sont également transposés dans la continuité des parcours biographiques, dans la fin de vie et face à la mort. En fonction de leurs origines et de leurs cultes, une partie des personnes immigrées souhaitent être inhumées dans leurs pays d'origine, c'est le cas par exemple de la majorité des Turques. L'inhumation en France peut représenter une appréhension pour s'assurer du respect des rites funéraires. C'est ce qu'exprime une représentante associative interrogée toujours dans l'exemple des personnes turques : "Je veux rejoindre mes ancêtres, ma famille en Turquie, je veux être enterré avec les autres là-bas. Voilà pour eux c'est leur terre, ils sont nés là-bas. C'est peutêtre... et puis d'être enterré tout proche [s'ils étaient inhumés en France] d'un non croyant, parce que dans l'islam cela se fait, cela ne se fait pas. Il y a des choses là-dessus " (Association 2 Angers, 22). Dans la fin de vie, certaines personnes peuvent partir en amont du décès pour être présentes dans le pays d'origine quand le décès surviendra. Pour exemple au sein des foyers ADOMA, les professionnels expriment que les personnes qui font le choix d'être inhumées au pays partent lorsqu'ils estiment que la fin est proche. "Ils le sentent qu'à un moment donné cela commence à s'épuiser et qu'ils n'arrivent de toute façon plus à gérer le quotidien ici, donc ils y retournent. Ou alors il suffit que cela soit un voyage au pays et puis qu'au final ils ne sont pas en capacité de revenir en France " (AS ADOMA, 35). Lorsque les personnes souhaitent être inhumées dans leurs pays d'origine, mais qu'elles décèdent en France, selon les situations et les origines, la personne a pu souscrire à une assurance ou contribuer à une caisse de solidarité entre pairs permettant la prise en charge des frais de rapatriement. L'entraide des proches est ici essentielle pour honorer la volonté du défunt et respecter les rites funéraires et les croyances de la personne. D'après une assistante sociale, "la communauté, oui, vraiment tout de suite elle prend contact avec nous, pour nous dire que tout est mis en place et que financièrement ils prennent en charge aussi » (AS ADOMA, 37). C'est donc difficile pour les intervenants quand la personne est isolée et qu'elle n'a pas fait part de ses souhaits, notamment vis-à-vis de la gestion du corps postmortem. Au sein du centre hospitalier de Cholet, 
le service social exprime ainsi que " pour une personne musulmane on se posait la question au niveau culturel de l'enterrement, dans un linceul, mais c'était un peu différent de ce qu'on connaissait donc on ne savait pas trop comment gérer ça, c'est une de nos collègues qui a donc géré ça [...] le corps ne doit pas être embaumé » (AS CH Cholet, 18). Au regard des éléments développés, les enjeux associés au fait de vieillir dans l'immigration sont perceptibles, ils s'ancrent dans la continuité des parcours, de la retraite jusqu'au décès et au sein d'espaces de vie pluriels pour les individus.

\section{Conclusion}

Analyser les conditions de vie et de vieillissement de la population âgée immigrée sur un département représente un défi complexe. Il s'agit d'éviter l'homogénéisation, tout en faisant part de situations ou de difficultés communes. Il faut souligner de nouveau que l'analyse est faite à partir des retours d'expériences des acteurs médico-sociaux, associatifs et politiques. Différentes limites et biais existent donc vis-à-vis de ce choix. C'est à travers le filtre de leurs interventions, de leurs représentations et des situations bien souvent les plus complexes ou difficiles que les acteurs ont fait part des enjeux du vieillissement des personnes immigrées. L'emploi dans l'étude des termes migrants âgés, personnes âgées immigrées, n'est pas neutre et les acteurs peuvent associer à ces catégories, différents profils d'individus (personnes originaires du Maghreb, d'Afrique subsaharienne) ou omettre certaines situations (les personnes originaires d'Europe : "eux, c'est différent»). La réalisation des entretiens a nécessité de réinterroger constamment les représentations des acteurs pour pouvoir ouvrir les discussions à l'hétérogénéité sociale et culturelle des migrants âgés.

Les exemples recueillis ne lèvent donc le voile que sur une partie des situations rencontrées en Maineet-Loire. Ils offrent cependant l'opportunité de prendre la mesure des problématiques que peuvent rencontrer les migrants dans l'avancée en âge et la multiplicité des dimensions en jeu. Au final, trois principaux enjeux différencient les expériences de l'avancée en âge des immigrés âgés d'après les propos des acteurs.

Premièrement, le fait que leurs parcours de vie soient inscrits dans des territoires pluriels, tissés entre le pays d'accueil et le pays d'origine. Si l'ensemble des personnes immigrées ne maintient pas des pratiques transmigratoires, une partie d'entre elles préserve des liens durables et forts entre les différents espaces géographiques et culturels qui composent leur vie. Le vieillissement et la dégradation de l'état de santé peuvent mettre à mal ces pratiques et ces liens. Les espaces de vie des individus se réduisant plus ou moins progressivement à mesure que leur situation évolue au regard de leur autonomie notamment. Pour Rafai et al. (2007), cela représente pour les immigrés âgés l'un des tournants biographiques, qui marque l'entrée dans la vieillesse. «Ces pratiques ne cessent vraiment que lorsque la personne âgée perd toute mobilité, et cette rupture apparaît de plus en plus comme un des principaux marqueurs de la « vraie » vieillesse, sinon de l'avènement du temps de la fin de vie » (Rafai et al., 2007).

Deuxièmement, les différences culturelles qui peuvent exister dans les modes de prises en charge de la vieillesse témoignent pour les acteurs de la place essentielle qu'occupent la famille et les proches dans le soutien apporté aux immigrés âgés. Bien que les familles non-immigrées ont un rôle aussi important dans le soutien aux personnes vieillissantes, le panel des solutions mobilisables par les personnes immigrées apparaît être plus restreint (non ou faible recours aux aides professionnelles et à l'accueil en EHPAD) et ce au regard des choix qu'elles font et/ou des contraintes qu'elles connaissent. Il faut cependant se prémunir, premièrement, d'une vision sacralisée de l'entraide familiale pour les immigrés âgés telle que l'a démontré Pian (2014) et deuxièmement les choix des personnes immigrées quant aux modalités de prise en charge de la vieillesse ne sont pas si éloignés des personnes françaises (Attias-Donfut et Gallou, 2006). L'analyse des parcours de vie des personnes immigrées est alors importante, car ceux-ci se sont ancrés dans différents territoires. Comme l'exprime Torres (2008), les personnes immigrées ont été en relation au cours de leur parcours avec des contextes culturels pluriels. La notion d'hybridité de l'expérience des migrants traduit cette idée, c'est « le fait que les migrants sont souvent exposés à des valeurs, des attitudes et des conceptions culturelles multiples et parfois contradictoires et qu'ils doivent opérer un arbitrage entre elles » (Torres, 2008). L'exposition à ces contextes, aux normes et valeurs qui les com- 
posent, est déterminante dans l'étude des situations des migrants âgés car les représentations de la vieillesse, du soutien apporté aux personnes âgées et de ce qu'est le bien-vieillir a pu évoluer au contact de la société d'accueil et tout au long de leur présence en France.

Troisièmement, vieillir dans un département où la population immigrée est faiblement représentée constitue un enjeu supplémentaire lié à leur « visibilité sociale». En effet, les interventions qui peuvent être qualifiées "d'à la marge » auprès de ces publics "invisibles", posent la question pour les acteurs et intervenants de leurs connaissances et de leurs formations aux enjeux singuliers que soulèvent ces accompagnements. Dans le cadre de la mission de l'ODAS, le conseil départemental faisait part de l'intérêt des évaluateurs de l'Allocation Personnalisée à l'Autonomie de bénéficier de formation sur la prise en charge des différences culturelles. Ces apports sont essentiels pour les professionnels afin de leur permettre de "développer leurs compétences culturelles tout en se gardant d'essentialiser les personnes, au risque de les enfermer dans une origine sociale ou culturelle stéréotypée et inopérante » (Wolff et Jovelin, 2015).

En définitive, appréhender les expériences de l'avancée en âge des personnes immigrées comprend de multiples enjeux tant sociaux que spatiaux qui existent tant au niveau individuel qu'à l'échelle des territoires d'actions des collectivités. En fonction des départements et des villes, la présence plus importante des immigrés âgés favorise la mise en œuvre d'initiative à leur attention (création de cafés sociaux dans certaines villes, inscription dans les schémas gérontologiques départementaux ${ }^{5}$, semaine bleue dédiée aux Chibanis à Montreuil en 2016). Des disparités dans l'offre d'actions et/ou de dispositions auxquels ils peuvent prétendre dans la vieillesse existent selon les territoires et de leur plus ou moins grande présence au sein de la population. Pour les personnes immigrées, l'avancée en âge s'ancre dans les espaces du quotidien, qu'ils fassent support ou contrainte dans la vieillesse, leurs pratiques spatiales apparaissant dans une partie des exemples recueillis comme restreintes ou limitées. Au vu de l'hétérogénéité sociale et culturelle des immigrés âgés, des

5. Dix départements français intégraient au sein de leur schéma gérontologique la question des immigrés vieillissants en 2008 selon une étude du Comité national des retraités et des personnes âgées (Bellot, 2008). recherches complémentaires sont nécessaires pour compléter et prolonger l'analyse qualitative menée auprès des acteurs. Dans cette perspective, saisir le bien-vieillir selon les propres représentations et perceptions des personnes immigrées âgées sur le département apparaît plus adapté pour comprendre les déterminants de leur bien-être dans la vieillesse. Cela appelle à la mise en œuvre d'entretiens auprès de ces publics dans le département pour analyser leurs expériences de vie, saisir leurs représentations culturelles de l'âge, de la place des personnes âgées, des solutions qu'ils envisagent face aux difficultés associées à la vieillesse. Ces entretiens doivent proposer une lecture de leurs parcours ancrée dans des territoires de vie différents pour mettre en lumière la diversité des espaces dans lesquels ils résident et interroger les possibles disparités qu'ils connaissent dans l'accompagnement du vieillissement. Ces résultats permettront de suivre l'avancée en âge de ces publics et de comprendre les expériences qu'ils connaissent dans la vieillesse à la lumière des espaces dans lesquels ils habitent et vivent.

\section{Bibliographie}

Attias-Donfut C., Gallou R., 2006. L'impact des cultures d'origine sur les pratiques d'entraide familiale, Informations sociales - CNAF, n ${ }^{\circ} 134$, vol. 6, p. 86-97.

Attias-Donfut C., 2012. Dépendance des immigrés âgés, une solitude accrue, in Gzil F., Hirsch E. (dir.), Alzheimer, Éthique et Société, ÈRES, coll. "Espace éthique », p. $458-471$.

BartKowiak N., 2008. L'accueil des immigrés vieillissants en institution, Rennes, Presses de l'école des hautes études en santé publique, $127 \mathrm{p}$.

Bellot C., 2008, Enquête sur les immigrés vieillissants, Comité National des Retraités et des Personnes Âgées, 31 p.

Conseil de développement de la région d'Angers, 2012. Riches de nos anciens, attentifs à leurs besoins, Angers, CDRA, 48 p. (non-publié)

Croguennec Y., 2012. Qui sont les immigrés âgés ? Infos migrations, $\mathrm{n}^{\circ} 34$, p. 1-4.

Dumont G.-F., 2005. Le siècle du vieillissement, Population $\varepsilon$ Avenir, no 671, vol. 1, p. 3 .

Gallou R., 2005. Les immigrés isolés : la spécificité des résidants en foyer, Retraite et société, no 44, p. 106-147.

Hamel C., Moisy M., 2013. Immigrés et descendants d'immigrés face à la santé, Paris, INED, vol. 190, 60 p.

Jacquat D., Bachelay A., 2013. Rapport d'information sur les immigrés âgés, Assemblée Nationale, Paris, 613 p. 
Marais J.-L., 2016. Histoire et mémoire de l'immigration en Anjou, 1965-1982, Hmia.fr [http://hmia.fr/histoire/lesperiodes/1965-a-1982].

Math A., 2012. Vieillesse immigrée, vieillesse harcelée : le contrôle par la résidence, Plein droit, nº 93, p. 3-7.

ODAS, 2014, Compte rendu déplacement Angers-Trélazé - document de travail. Observatoire national de l'Action Sociale (ODAS), 17 p. (non publié).

Paillé P., Mucchielli A., 2008. L'analyse qualitative en sciences humaines et sociales, Paris, Armand-Colin, coll. « Sciences humaines et sociales », $315 \mathrm{p}$.

Pian A., 2014. Désacraliser la solidarité familiale. Quand le cancer touche des immigrés âgés, Norois, n 232, vol. 3, p. 67-80.

Rafaï K., Mantovani J., Duchier J. et al., 2007. Le vieillissement des migrants, situation en Midi-Pyrénées, Rapport ORSMIP et INSERM U 558, 146 p.
SAmaoli O., 2012. La vieillesse des immigrés. La fin du voyage!, Écart d'identité, n $\mathrm{n}^{\circ} 120$, p. 55-61.

Soumeur-Méreau E., 2014. Le vieillissement des populations immigrées, RésoVilles, 96 p.

Torres S., 2008. L’ère des migrations, Retraite et société, n 3 , vol. 55, p. 15-37.

Warnes A., Kellaher L., Friedrich K. et al., 2004. Europe The diversity and welfare of older migrants in Europe, Ageing and Society, $\mathrm{n}^{\circ}$ 3, vol. 24, p. 307-326.

Wolff V., Jovelin E., 2015. L'accompagnement social et sanitaire des personnes âgées immigrées, Hommes \& Migrations, $\mathrm{n}^{\circ} 1309$, p. 97-104. 